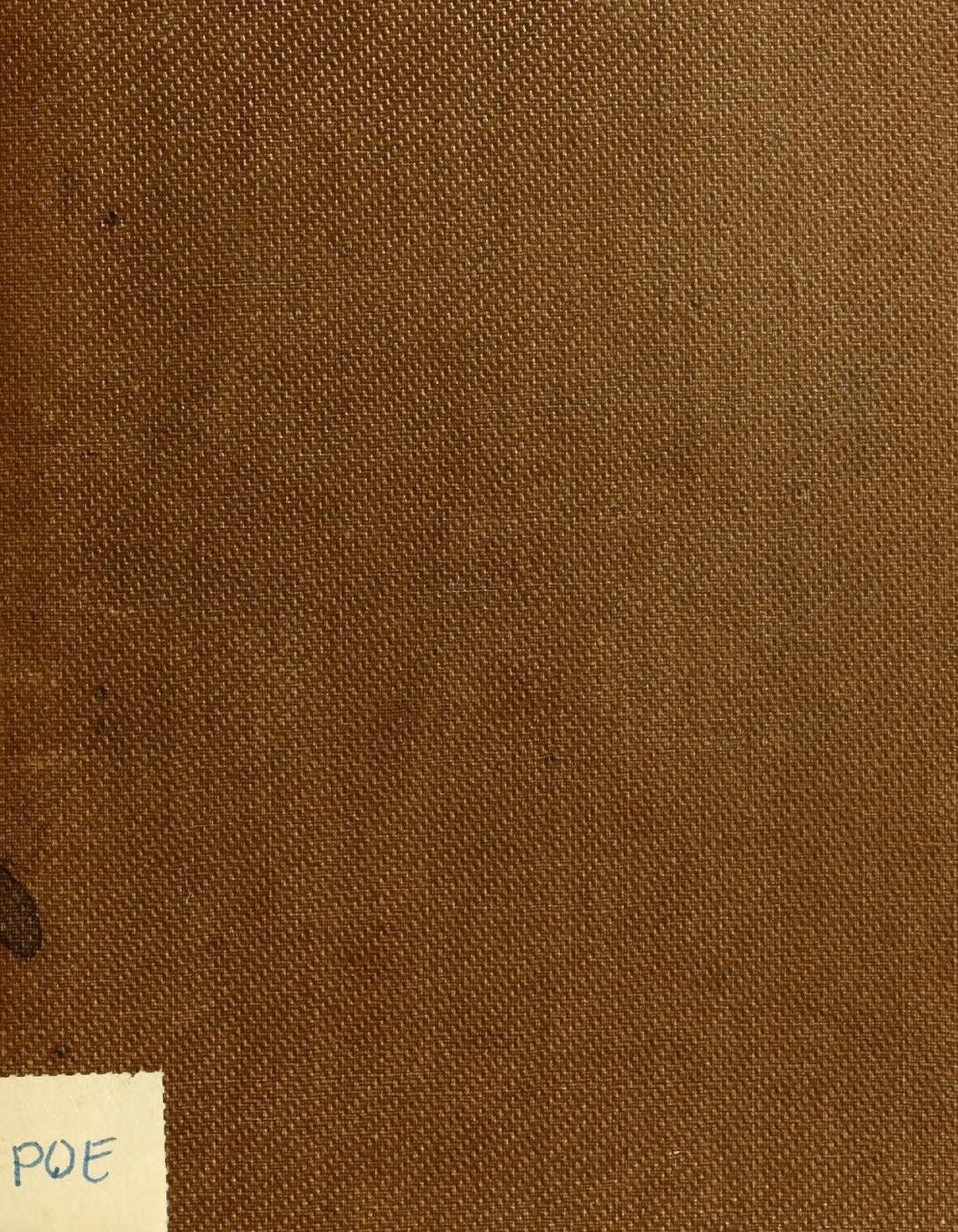




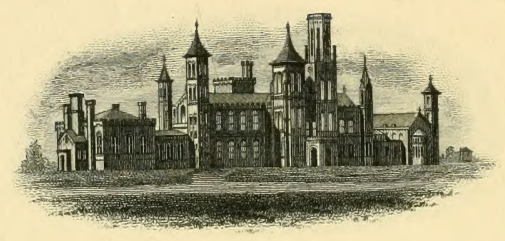

\title{
COLLECTION \\ OF
}

WILLIAM SCHAUS

(D)

\author{
PRESENTED
}

TO THE

NATIONAL MUSEUM

MCMV 




\section{CENTURIE DE LÉPIDOPTÈRES}

DE L'ILE DE CUBA,

CONTENANT

LA DESCRIPTION ET LES FIGURES COLORIÉES

DE

\section{CENT ESPĖCES DE PAPILLONS}

NOUVEAUX OU PEU CONNUS,

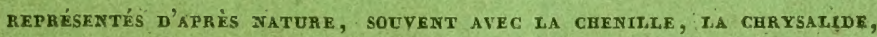
ET PLESLEUR DÉTATLS MIGROSCOPIQUES;

\section{pate PH. POEY,}

MEAMRE DE IA SOCZÉTÉ ENTOMOLOQIQUE DE FRAYCE, ET NEMBRE CORRESPONDANT DE IA SOCTÉTÉ ROYALE ET PATRTOTIQUE DE LA HAVANE.
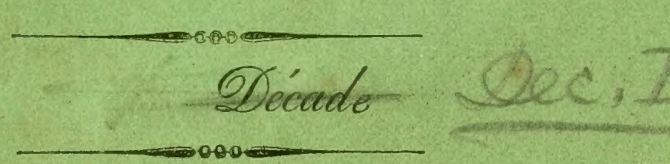

ON SOUSCRIT A PARIS,

A LA LIBR AIBIE GREGQUE-LATINE-ALLEMANDE ET ANGLAISE

DE J. ALBERT MERCKLEIN,

RUE WES BEAUX-ARTS, $\mathbb{N}^{\circ} \mathrm{I}$.

M DCCC XXXII. 

Gen.Callidryas, Bden
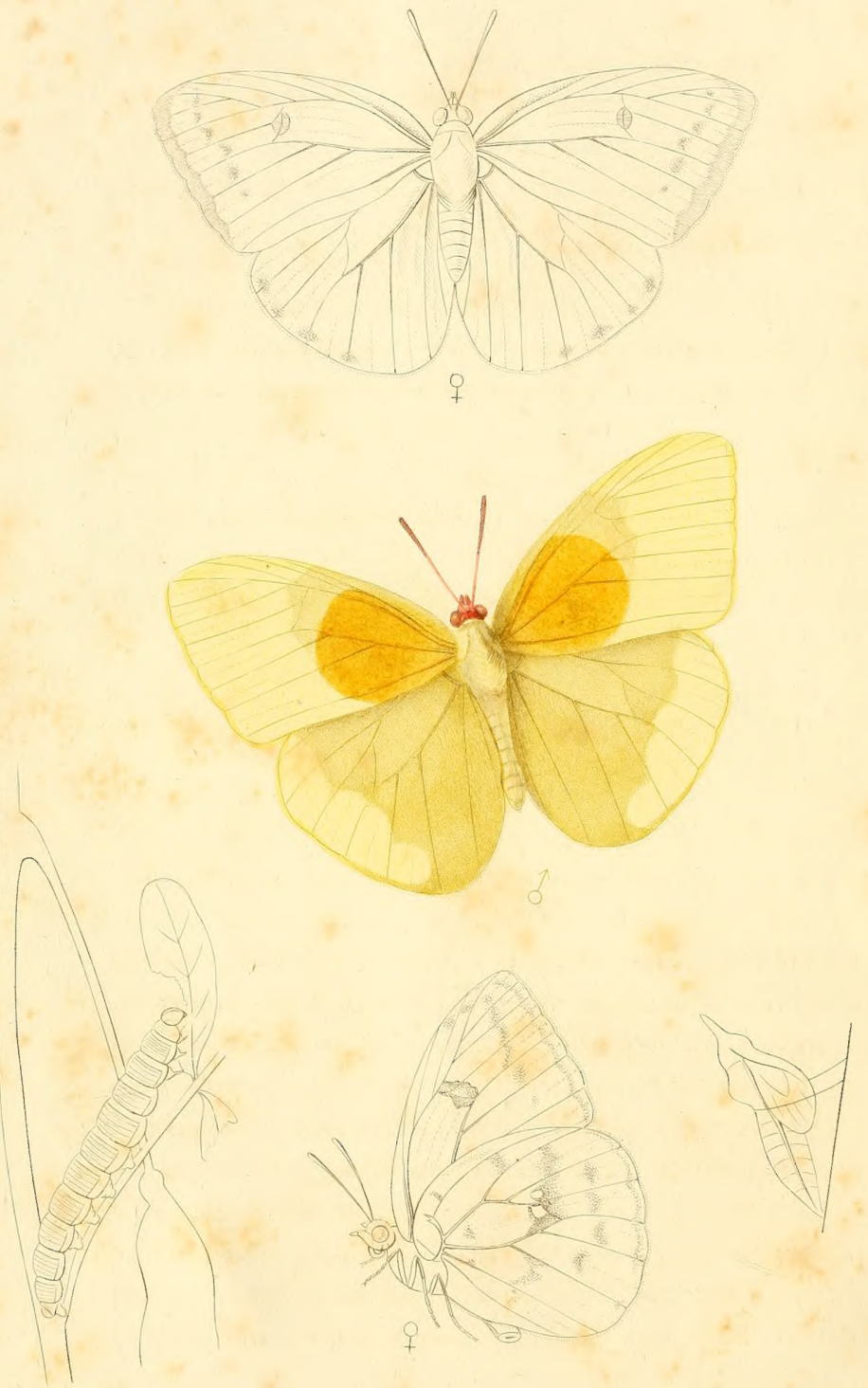

(O)RBIS 


\section{NUM.}

\section{Callidryas Orbis, Poey.}

Alæ integerrimæ rotundatæ flavæ; mas, supra et antice macula magna flavissima; fæmina, posticarum punctis marginalibus, anticarum puncto medio non ocellato, margine lunulisque fuscis; subtus lunulis ferrugineis, et posticis puncto gemino argenteo.

Callidryade Orbis. - Ailes très-entières et jaunes; le dessus du mâle présentant, sur les ailes supérieures, une grande tache orbiculaire plus foncée; la femelle ayant le bord des inférieures entrecoupé de brun, et les supérieures avec une bordure marginale, une tache centrale entière, et des lunules brunes; le dessous avec des lunules ferrugineuses, et deux points argentés aux inférieures.

\section{DESCRIPTION.}

Voyez les deux sexes dans la planche ci-jointe, avec la chenille, la plante qui lui sert de nourriture, et la chrysalide.

Les antennes, le dessus de la tête, les palpes supérieurs et le dessous des inférieurs, rouges; ceux-ci de forme ordinaire dans le mâle, avec tous les articles également recouverts d'écailles; la femelle ayant au contraire le dernier article cylindrique, horizontal, presque nu et tout rouge. Les ailes du mâle ne présentent aucune touffe de poils, ni aucune autre marque distinctive du sexe, outre les couleurs, si ce n'est qu'elles sont plus prolongées vers l'angle anal. 
Lesindividus de ce sexe ont, sur les ailes supérieures, une tache arrondie d'un jaune foncé, ne touchant pas la base et n'atteignant pas la côte; la femelle est tout entière de cette dernière couleur. Le dessous, plus pâle dans le mâle, porte l'empreinte de la bordure marginale du dessus, et brille d'un léger reflet pourpré. Les premières ailes ont, au centre, un gros point ferrugineux; les secondes deux points argentés, dont l'un est placé sur la ligne qui ferme la cellule discoïdale, l'autre en dehors et au dessus.

La chenille, d'après un dessin médiocrement soigné que j’ai fait sur les lieux, est d'un vert assez foncé, avec une bande transverse d'un vert tendre sur chaque anneau. La Chrysalide est verte, avec les extrémités couleur de rose, et les nervures jaunes. La tête, prolongée en corne, n'est point arquée, comme dans la C. Eubule.

OBSERVATIONS.

Le mâle diffère de tous ses congénères par la tache orbiculaire des ailes supérieures; car, dans l'Hilaria de Cramer et dans quelques autres espèces voisines, toute la base des quatre ailes est jaune. La femelle diffère de l'Eubule de Linné, et de plusieurs espèces américaines, par le point discoïal des supérieures, qui est entier, au lieu d'être divisé ou semi-ocellé. Elle diffère de la femelle d'Hilaria et de quelques autres espèces orientales, par les taches nacrées du dessous, qui ne sont point cerclées de ronge.

M. Donovan, dans son bel ouvrage sur les Insectes de l'Asie, a figuré une espèce de la Nouvelle-Hollande, qui ressemble beaucoup à la nôtre. Il la rapporte à la Pomona de Fabricius, qui la décrit cependant comme ayant les ailes presque anguleuses et blanches. M. Donovan qui, plus que tout autre, est à portée de vérifier la nomenclature de ce 
célèbre entomologiste, aurait dî nous donner quelques explications sur ce sujet.

La Danaide Sennce de Linné n'est autre chose que l'Éubule du même auteur, et Marcellina de Cramer n'en est qu'une variété.

HISTOIRE.

Cette espèce se trouve dans l'île de Cuba, où elle est rare, surtout le mâle. Je l'ai cependant élevée quelquefois, ainsi que l'espèce, très-commune, nommée Eubule; ce que jecrois utile d'observer ici pour ne point laisser de doutes sur la formation des deux espèces.

Linsecte porte, dans l'inaction, les antennes contiguës; son vol est ferme comme celui de la Coliade du Nerprun (Rhamni, Lin.). Il se montre volontiers daus les jardins cultivés.

La chenille se nourrit de feuilles du poinciana élégant (Poinciana pulcherrima, Lin.), nommé vulgairement Guacamaya à la Havane.

Le nom d'Orbis fait allusion à la tache arrondie des ailes supérieures, dans le mâle.

Avril 1832 . 


Gen Malitea, fint
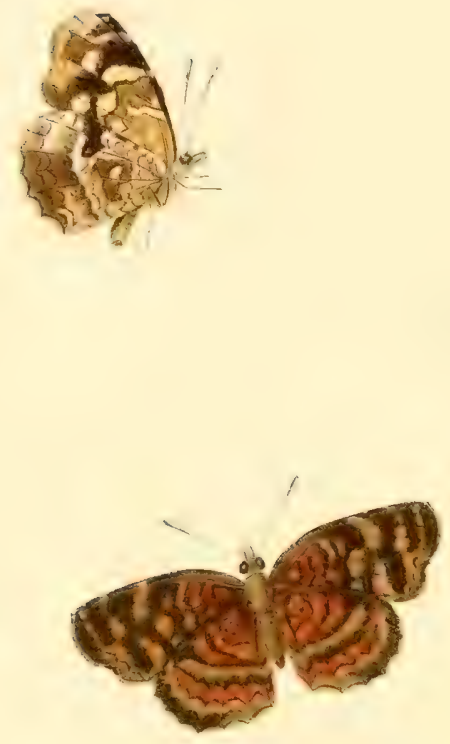

IIRISIII 


\section{NUM.}

\section{Melitea Frisia, Poey.}

Alis subintegris, nigris; anticis supra fulvomaculatis, posticis fasciatis; antice subtus fulvæ, macula magna fusca; posticie pallescentes, lineis undatis strigaque punctorum nigrorum.

Mízitmée Frisia. - Ailes presque entières, noires, avec des taches aux supérieures, et des bandes aux inférieures, fauves. Le dessous est de cette dernière couleur, avec des teintes brunes; les inférieures plus pâles, avec des lignes transverses et une rangée de points noirs.

\section{DESCRIPTION.}

Voyez les deux sexes, planche ci-jointe.

Les ailes supérieures sont échancrées sur le bord extérieur, vers l'angle interne, avec deux cellules à frange jaune sur l'échancrure, et une autre peu distincte vers le sommet. Les points noirs du milieu des inférieures sont ordinairement aveugles, quelquefois ocellés ou cerclés de jaunàtre.

\section{OBSERVATIONS.}

Les espèces les plus voisines sont au nombre de six, dans les auteurs; mais elles peuvent être rapportées aux trois stivantes: 
I. Tharos., Dr, 1. $21: 5.6$.

Tharossa, God., Enc. Arg. 61 .

Gorgone, Hüb. Sam. exot., tom. i.

Euclea, Bergs., $79:$ I. 2.

2. Morpheus, Fab., Sp. Pap. 278. Ent. syst. Pap. 479.

IMorphea, God., Enc. Arg. 60.

Cocyta, Cram., I0I : A. B.

3. Liriope, Cram., I. : C. D.

Ces espèces diffèrent de la Mélithée Frisia en ce que le fauve domine dans les unes, en dessus, et les autres offrent, sur les ailes inférieures, une série de points noirs, qui forment ici une bande continue. La teinte noire du dessous des premières ailes est aussi différemment placée, et annonce l'absence d'une tache fauve sur l'antre surface.

HISTOIRE.

Cette espèce est très-commune dans l'île de Cuba, surtout vers la côte du Nord. Linsecte parfait habite les prairies et les lieux cultivés, où il voltige sur des plantes basses, en s'élevant très-peu au-dessus de terre.

Le nom de Frisia rappelle la femme de Caupolican, le héros du poème espagnol d'Ercilla, intitulé La Araucana, ouvrage de premier ordre, qui abonde en beautés de tous genres, malgré les irrégularités que Voltaire a relevées dans ses critiques.

Avril I 832. 

Gen. Eumenia, God
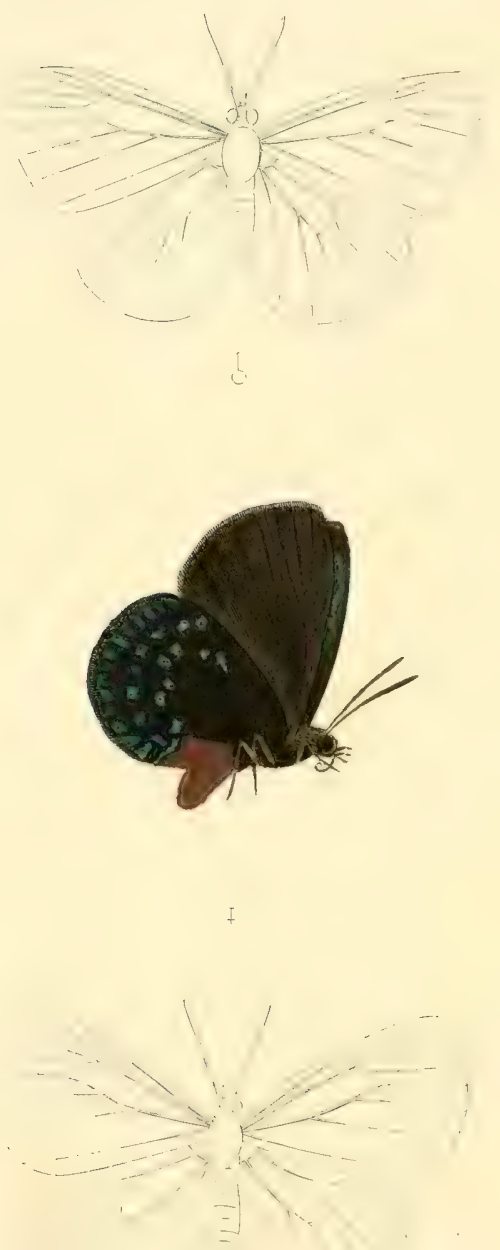

ATTATIA 


\section{NUM. 3}

\section{Eumenia Atala, Pocy.}

Eum. Toxea (God.), Guérin, Icon., pl. 8o, fig. 3.

Le mâle.

Alis integerrimis, rotundatis, nigris; disco supra, et maculis marginalibus posticarum cærtleo-nitentibus; subtus triplici macularum serie, nitide cærulescentium, interna inæqualiter disposita, maculaque magna rubra ad marginem interiorem. Abdomine rubro, basi fusco; fimbria nigra.

Euménie Atala. - Ailes entières, arrondies, noires, avec: le disque, en dessus, et le bord des inférieures d'un bleu luisant; celles-ci ont, en dessous, trois séries de taches bleues et brillantes, l'intérieure irrégulière ou non parallèle, et une grande tache rouge sur le bord interne. Abdomen rouge, hormis la base, qui est brune; frange noire.

DESCRIPTION.

Voyez les deux sexes dans la planche ci-jointe.

Les antennes forment une massue qui grossit insensible. ment depuis la base; elles sont bien représentées dans l'Iconographie du Règne animal, ouvrage éminemment instructif, publié par M. Guérin. Les palpes sont écartés, comprimés, horiznntaux, le deuxième article très-long. Les yeux, cer- 
clés en arrière par des écailles luisantes, comme dans les espèces du genre Polyommate, auprès duquel celui-ci doit ètre placé.

Le dessus des ailes est d'un bleu brillant, depuis la base jusqu'au-delà du milieu : cette teinte est beaucoup moins étendue dans la femelle.

\section{OBSERVATIONS.}

On ne connaît, jusqu'à présent, qu'une autre espèce du même genre, nommée Minijas par Hübner dans le Saml. exot., tom. I, et Toxea par Godart, Encycl. méth., tom. IX d'Hist. nat., pag. 826 ; elle a servi de type à Godart pour former le genre Euménie. L'espèce que je décris en est si voisine que je l'ai d'abord confondue avec la première; et M. Guérin, qui l'a reçue de moi pour la figurer dans son Iconographie, a partagé mon erreur en la rapportant à l'Eum. Toxea de Godart. Mais, en l'examinant plus attentivement, on remarque entre elles les différences suivantes :

\section{Eum. Minijas, Hüb. \\ Toxea, God.}

Eum. Atala.

x. De taille un peu plus grande.

2. Abdomen noir, avec l'extrémité, seulement, rouge.

3. Frange toute blanche.

4. Palpes un peu plus larges, vus de côté.

5. Teinte brillante du dessus des ailes plus étendue.

6. Les points marginaux des inférieures, en dessus, plus grands.

7. Rangée interne des taches du dessous parallèle aux deux autres, sur une ligne qui aboutit au tiers de la côte des ailes inférieures.

8. Tache rouge du bord interne des inférieures moitié plus petite, ce qui la fait paraitre abdominale.

9. Patric : L'Amérique méridionalc. r. Un peu plus petite.

2. Abdomen rouge, arec la base, seulement, noire.

3. Frange entièrement noire.

4. Ces mêmes palpes plus petits.

5. Cette méme teiute moins étendue, surtout dans la femelle.

6. Ces mêmes points plus petits.

7. La même rangée placée sur une ligne irrégulière, et aboutissant au milieu de l'aile.

8. Cette même tache plus grande et s'avançant vers la base de l'aile.

9. Patric: L'ile de Cuba. 
C'est à tort qu'Hübner a représenté son Minijas avec la base des inférieures plus claire que le reste de l'aile. Le fond est partout égal dans les deux espèces, quoique plus foncé dans Atala.

\section{IIISTOIRE.}

Cette espèce n'est pas commune. Je n'en ai eu que trois individus, pris dans le même lieu, par un enfant qui les portait dans sa main, sans les retenir, et sans que l'insecte essayât de s'échapper; ce qui fait croire que son vol est lourd, à moins qu'ils ne vinssent tous trois d'éclore. Depuis j'en ai vu encore cinq à Paris, provenant toujours des environs de la Havane, et qui ont appartenu à M. Rippert.

Avril 1832 . 


Gen Hesperia, Fab
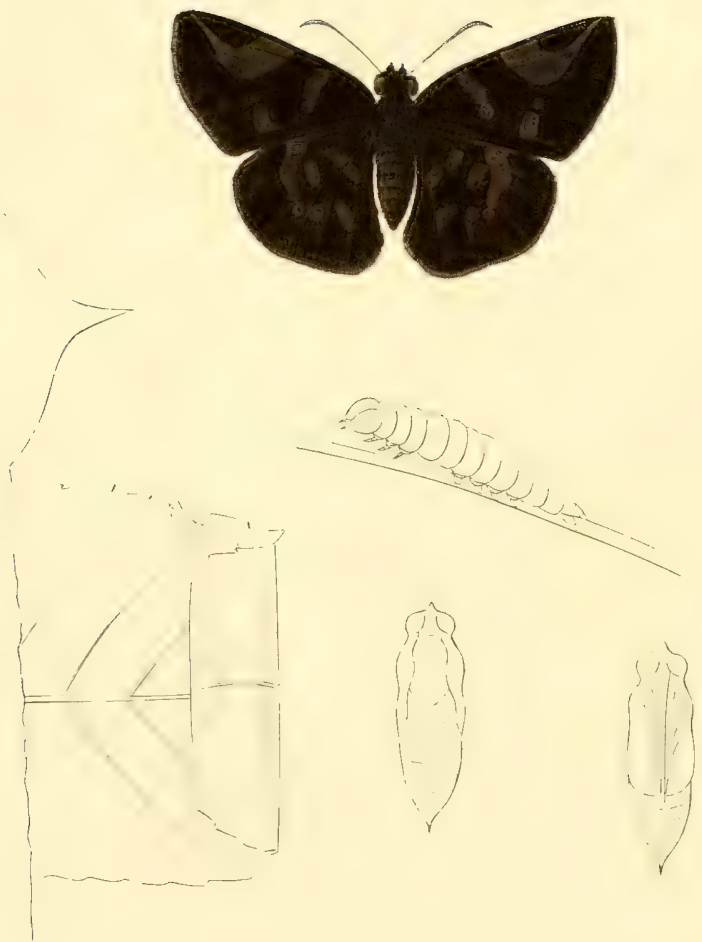

PAPINIANTS. 


\section{NUM.}

\section{Hesperia Papinianus, Poey.}

Antennæ uncinatæ. Alæ integerrimæ, fuscæ, supra punctis plurimis pallidioribus, anticarum lunula apicali magna. Subtus fusco-fasciatæ, posticarum apice cæruleo-cinarescente.

Hespérie Papinien. - Extrémité des antennes recourbée. Ailes entières, brunes, avec plusieurs taches plus pàles en dessus, et une lunule plus grande vers le sommet des supérieures. Le dessous traversé par des bandes plus ou moins claires, avec l'extrémité des inférieures pointillée d'un cendré bleuâtre.

\section{DESCRIPTION.}

Voyez le mâle, płanche ci-jointe, avec la Chenille et la Chrysalide, ainsi que la plante, dont je n'ai représenté que l'épine, faute d'en avoir conservé autre chose, si ce n'est toutefois le nom vulgaire, que je rapporterai plus bas.

Les palpes de linsecte parfait ont le dernier article trèscourt, presque entièrement caché sous les écailles environnantes. La massue des antennes est ferrugineuse en dessous. Tout le corps brun.

La femelle doit ètre beaucoup plus pàle, avec des taches plus grandes, d'après ce qu'on observe dans les espèces voisines.

La Crennure est verte, avec la tête d'un brun rougeâtre.

La Chrysalide est noire, mais couverte d'une poussiere blanche dont la Chenille a rempli la feuille sous lacquelle elle s'est dépouillée. 
Cette espèce pouvait se rapporter à l'Hespérie Thrasibule de Fabricius; mais la phrase trop courte de cet auteur s'applique également à plusieurs autres espèces. C'est d'après la description plus étendue de M. Latreille, dans l'Encyclopédie méthodique, que je laisse le nom de Thrasibule à une espèce du Brésil, figurée dans Hübner, Saml. exot. Schmet., tome I, laquelle a de grands rapports avec la nôtre, mais qui en diffère par les caractères suivants, observés dans les mâles :

Hesp. Thrasibule, Fab.

Hesp. Papinien.

Traso, Hüb.

r. Massue des antennes plus étroite.

2. Couleur des ailes plus foncée, d'un brun violet un pen changeant.

3. Tache du milieu de la seconde cellule interne des ailes supérieures toujours bifide ou presque double.

4. Frauge blanche sur une cellule du sommet des supérieures, qui se terminent par un angle plus prononcé.

5. Taches plus petites; plusieurs oblitérées aux inférieures.

6. Le dessous, toujours d'un brun clair au sommet des inférieures.

7. Patrie : Le Brésil.
I. Massue des anteunes plus épaisse.

2. Ailes plus grises, se rapprochant de la femelle de Thrasibule

3. Cette même tache bien entièrc et arroudie.

4. Point blanc à la frange des supéricures; sommet moins anguleux.

5. Taches graudes, autant que celles de la femelle de Thrasibule, et très-dis. tinctes.

6. Bord des inféricures, en dessous, toujours parsemé de molécules d'un bleu cendré presque blanc.

7. Patrie : Cuba.

L'étude de cette espèce m'en a fait découvrir deux autres très-voisines, et qui sont encore nouvelles. En voici les noms et la description :

I'Hesperia Ulpianus, Poey.-L'Hesp. Ulpien a la même coupe d'ailes, et appartient au mème groupe que Papinicn, 
dont elle a aussi la taille. Le màle est noir en dessus, sans taches, avec tout l'angle interne des supérieures largement mais faiblement pointillé de molécules grises, qui disparaissent insensiblement vers le sommet, dépourvu de lunule. Les inférieures, depuis l'extrémité jusqu'au-delà du centre, offrent les mêmes atomes cendrés, excepté sur une bande étroite de la couleur du fond, placée près du milieu de l'aile. Le dessous est brun, sans aucune bande, avec toute la surface des ailes inférieures couverte d'atomes cendrés, d'un bleu clair, plus nombreux du milieu au bord interne. Corps et palpes gris en dessous. Je l'ai eue de M. Gory, qui l'a reçue du Brésil. Je n'ai pas vu la femelle.

$2^{\circ}$ Hesperia Labeo, Poey.-L'Hesp. Labéon est encore du même groupe, et à peu près de même taille. La fèmelle est d'un brun noir, beaucoup plus foncé que celle de Thrasibule, avec les mêmes taches, peu distinctes à cause de leur intensité, et formant des bandes continues; la lunule du sommet existe très-incomplètement, car elle n'est indiquée que par des molécules blanchàtres, semblables à celles qui bordent les quatre ailes, et que le moindre contact peut enlever. En dessous, cette lunule est remplacée par un espace triangulaire, couvert d'atomes blancs, serrés et distincts; mais l'angle anal n'en contient qu'un très-petit nombre. L'extrémité des antennes est moins crochue que dans les espèces voisines. Elle est de la collection de M. le colonel Feisthamel. Je n'ai pas vu le mâle. Elle habite...

Ces quatre espèces, et plusieurs autres qui ont la méme coupe d'ailes avec les antennes crochues, formeront sans doute un nouveau genre parmi les Hespéries.

IISTOIRE.

Cette espèce est commune; j’en ai rapporté sept individus, parmi lesquels je n'ai pas eu une seule femelle. Ia Chenille 
se nourrit de feuilles d'un arbre nommé, dans le pays, Ayuda, mot espagnol qui rappelle peut-être une vertu purgative dans la plante. Le tronc est couvert d'épines courtes, à large base, comme celle que j’ai fait représenter. La Chenille en roule les feuilles pour se cacher et se préserver de ses ennemis.

Les noms romains ont été prodigués aux Hespéries : pour moi , je me fais un plaisir de dédier cette espèce au prince des jurisconsultes, mis à mort par un tyran au milieu de sa carrière, et mutilé, 300 ans après sa mort, par les barbares compilateurs du règne de Justinien.

Avril 1832 . 

Gen. Amphonyx, l'ocy.

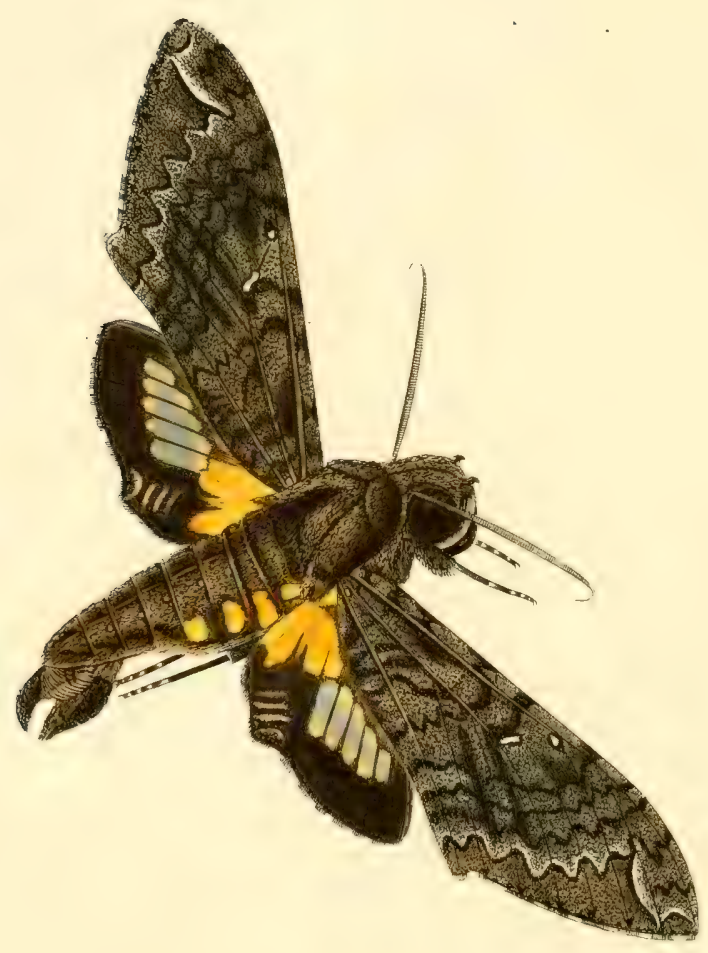

D) UYPONCTHEI. 


\section{NUM.}

\section{Amphonyx Duponchel, Poey.}

CHA RACTERES GENERIS.

Genus Anphonyx, Poey. - Differt a Sphingibus Linnæi et Latreillii ab ultimo articulo palporum, longiore, nudo, valido, extra caput distincto. Tunc capitis facies bicornis, sive cornubus duobus incurvis ante oculos.

Genre Amproxyx.--Il ne diffère du genre Sphinx de Linné et de Latreille que par le dernier article des palpes, qui est plus long, nu, corné, fortement implanté sur l’article précédent, et saillant au devant de la tête; ce qui la fait paraitre armée d'une double corne.

CIARACTERES SPECIEI.

Alis integris fuscis; anticis supra atomis viridibus irroratis, maculis plurimis transversis, lunulaque longitudinali undata versus apicem, nigris; puncto baseos punctisque duobus in medio albis; posticarum supra omniumque subtus basi aurantio, medio vitreo. Abdomen maculis trium parium rufis, subtus album.

Ayphonyx Duponcinel. - Ailes entières brunes; les supérieures convertes, en dessus, d'atomes verts, avec plusieurs 
bandes transverses, et une lunule longitudinale, tortueuse, vers le sommet, noires; un point blanc sur la base et deux sur le milieu; les inférieures vitrées au centre, et orangées à la base, ainsi que vers la racine des quatre ailes en dessous. Abdomen blanc de ce côté, avec trois taches latérales, roussâtres en dessus.

DESGRIPTION.

Voyez le mâle, planche ci-jointe.

Le crin qui part de la base des ailes inférieures est unique dans le mâle, et retenu par un frein implanté sur la côte des antérieures, tandis qu'il est multiple dans la femelle, et appuyé sur une éminence de ces mêmes ailes vers le bord interne. Les crochets du mâle sont extrêmement développés. La langue, très-longue, déborde toujours les palpes, lors même qu'elle est bien roulée.

Sa taille ne varie pas; la femelle est généralement plus pâle et un peu plus grande.

Le dessous est brun, avec la base des ailes orangée; cette teinte forme aux supérieures une large ligne longitudinale au dessous de la nervure centrale; elle est interrompue à son origine sur l'espace parcouru par le crin.

OBSERVATIONS.

Sur le genre. - Le mot Amphonyx vient de deux mots grecs : d'àusi, préposition, qui, en composition, signifie de chaque cóté, et d'òvus, uxos, ongle; pour exprimer le caractère indiqué ci-dessus. Il se décline en latin, Amphony $x$ ychos; pluriel , Amphonyches; nom masculin dans les deux langues.

Les palpes des Sphinx sont composés de deux articles distincts; le troisième existe probablement dans toutes les 
espèces; mais il est si petit qu'il a échappé à mes recherches dans deux Sphinx de belle taille, le Convolvuli et le Vitis de Linné. Je n'ai pu le voir, à l'aide d'une forte loupe, que dans le Sph. Ficus (Lin.); sa forme est ovoïde; sa grandeur ne dépasse pas le tiers d'un millimètre.

La longueur de cet article offrait donc, dans notre genre, un caractère très-distinct pour séparer du genre Sphinx, si nombreux en espèces, un petit nombre d'insectes. Ces deux onglets pourraient être considérés comme des moyens de défense, si toutefois un être aussi faible pouvait se défendre autrement que par la fuite. Les Sphinx Antaus, Dr., et Cluentius, $\mathrm{Cr}$., sont du même genre : ce dernier n’a pas les inférieures vitrées.

Sur l'aspìce. - L'Amp. Duponchel ne peut être confondu qu'avec l'espèce suivante :

Antæus, Dr. 2. 25 : 1.

Jatrophae, Fab., Syst. Sph. 8. Ent. syst. Sph. 22.

Hydlaspus, Cram., I $8, \mathbf{A}$.

Medor, Cram., 394, A.

L'Antceus, Dr., que Samenstel et Müller ont confondu avec le Carica, Lin., a un pouce de plus d'envergure. Il est plus noir ou privé de molécules olivâtres, ainsi que d'échancrure sur le bord extérieur des ailes supérieures vers l'angle interne; du moins ce caractère n'est pas aussi prononcé que dans notre espèce. Le faisceau qui garnit la base des ailes inférieures est composé, dans celle-ci, de 25 crins, tandis que j'en ai compté dans l'autre plus de quarante. Jo n'ai pu répéter l'opération sur plusieurs femelles.

HISTOIRE.

Cette espèce se trouve abondamment aux environs des habitations, dont elle ne craint pas de s'approcher, notam- 
ment dans le jardin botanique de la Havane. Elle vole le soir, an coucher du soleil, autour des fleurs d'une espèce de nictane, appelée dans le pays Maravilla (Merveille), où l'insecte parfait va puiser sa nourriture, ainsi que sur les fleurs de l'Ahouai des Antilles (Cerbera Tevetia, Lin., et vulgairement, dans le pays, Cabalonga). Quand l'insecte est vivant, il exhale une forte odeur de musc; particularité que je n'ai olsservée dans aucun autre Sphinx, et qu'il doit peutêtre à quelque fleur.

J'ai dédié cette espèce à M. Duponchel, auteur d'une Monographie des Érotyles, et continuateur de l'ouvrage de Godart sur les Lépidoptères de France. Ses connaissances dans cette partie de l'histoire naturelle, et le talent qu'il a mon tré dans cette continuation, doivent inspirer une grande confiance aux souscripteurs de deux nouveaux ouvrages qu'il a récemment annoncés : l'un servant de supplément à l'Histoire des Lépidoptères; l'autre destiné à offrir l'Iconographie des Chenilles d'Europe.

J'ai suivi MM. Latreille et Godart (Enc. méth.) dans l'usage de ne point écrire les noms propres au génitif.

Avril 1832. 



\section{Gen. Noctua, Lin .}
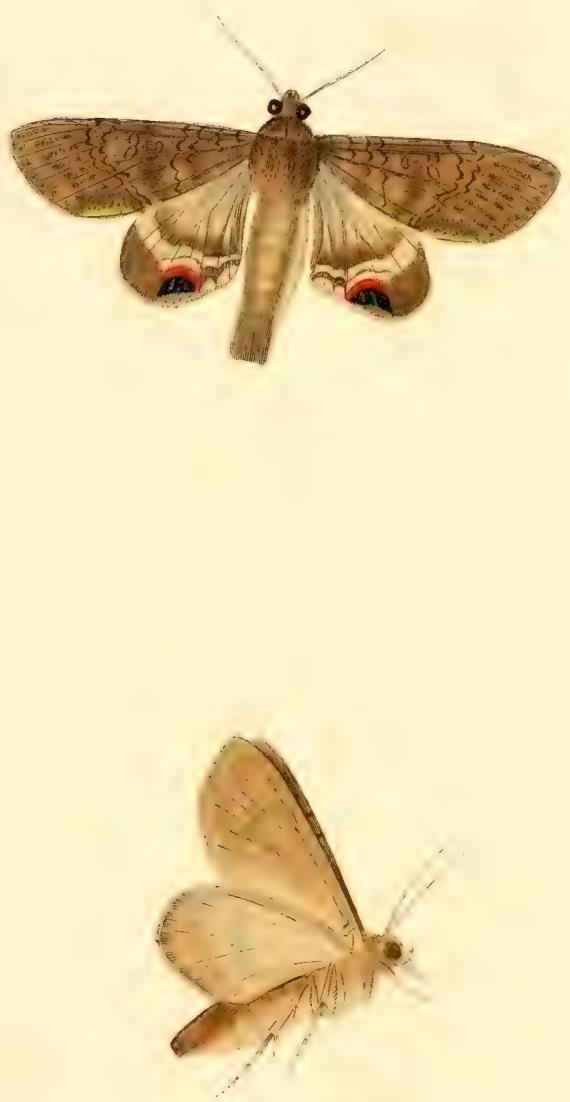

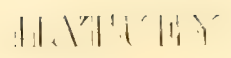




\section{NUM.}

\section{Noctua Hatuey, poey.}

Alæ anticæ fusco-rubræ, lineis transversis nigris, macula flava ad angulum interiorem, atomis cæruleo-micantibus circumdata; posticæ fuscæ, basi fasciaque flarescentibus, macula semi-ocellata, ad apicem nigra, iride rubra, pupillis geminis nitenti-cæruleis.

Noctcelle Hatcex.-Ailes supérieures d'un brun rougeâtre, avec des lignes transverses noires, et une tache jaunàtre vers l'angle interne des supérieures, surmontée de molécules bleues et luisantes; les inférieures brunes, avec la lase et une bande jaunàtres, l'extrémité ayant une tache noire, surmontée d'un iris rouge, et deux points au centre d'un bleu luisant.

\section{DESCRIPTION.}

Voyez le mâle, planche ci-jointe.

Antennes très-simples; crin des inférieures unique, et arrèté par un frein, qui part de la seconde cellule interne. Chaque article des tarses est brun au milieu, et blanc sur les deux bouts. Ine touffe serrée de poils à l'extrémité de l'abdomen. Je n'ai pas vu la femelle.

\section{OBSERVATIOY.}

La coupe d'ailes de cette espèce est assez singulière, et la tache ocellée des inférieures est bien remarquable dans une 
Noctuelle. Je ne connais point d'espèce voisine de celle-ci qui puisse entrer dans le même groupe.

HISTOIRE.

Le papillon est très-rare. Je ne l'ai pris qu'une seule fois dans le cafétal de MM. Gonzalo Alfonso et Foudras, à $L a$ gunillas, environ so lieues S.-E. du port de Matanzas.

Avril 1832 . 

Gen Erebus, Iat
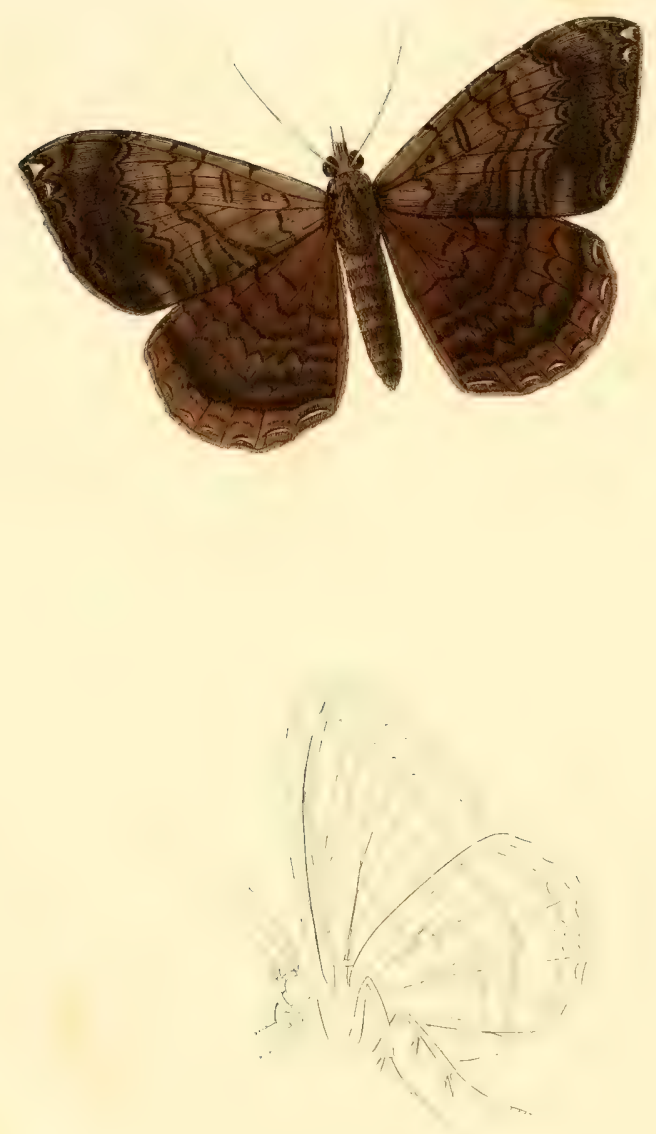

RENGTS 


\section{NUM.}

\section{Erebus Rengus, poey.}

Alis fuscis, lineis transversis nigris, anticis supra angulo interiore late nigro, maculisque dilutioribus, omnibus punctis marginalibus albis, obsoletis, primo apice anticarum distincto.

Erebus Revgus.—Brun, avec des lignes noires transverses; l'angle interne des ailes supérieures noir , avec des taches plus claires; quelques taches marginales blanches, peu prononcées, celle du sommet des antérieures bien distincte.

\section{DESCRIUTION.}

Voyez la figure ci-jointe; la $q$ ?

Les antennes sont légèrement pectinées; le crin est triple, et retenu par un frein, qui part de la deuxième cellule interne. L'abdomen manquait chez l'individu que j’ai fait figurer; mais le caractère tiré du crin indique une femelle; car il est constamment simple dans les Lépidoptères mâles, et triple dans les femelles du G. Erebus.

Le fond est brun en dessous, tout pointillé de gris, avec deux rangées de traits blancs.

\section{OBSERVATION.}

La teinte noire qui couvre l'angle interne des ailes supérieures suffit pour distinguer cette espèce de toutes celles du même genre. Il ne faut point négliger d'examiner le dessons dans la détermination de ces espèces; peu d'entre elles 
ont un aussi grand nombre de lignes transverses sur cette surface.

HISTOIRE.

Cette espèce est rare, car je ne l'ai prise qu'une seule fois pendant plusieurs années que j’ai chassé dans le pays. Comme toutes celles du même genre connues à la Havane sous le nom de Brujas (Sorcières), elle entre dans les maisons, et se cache sous les toits.

Avril 1832. 

Gen. Mastigophorus Mocy.
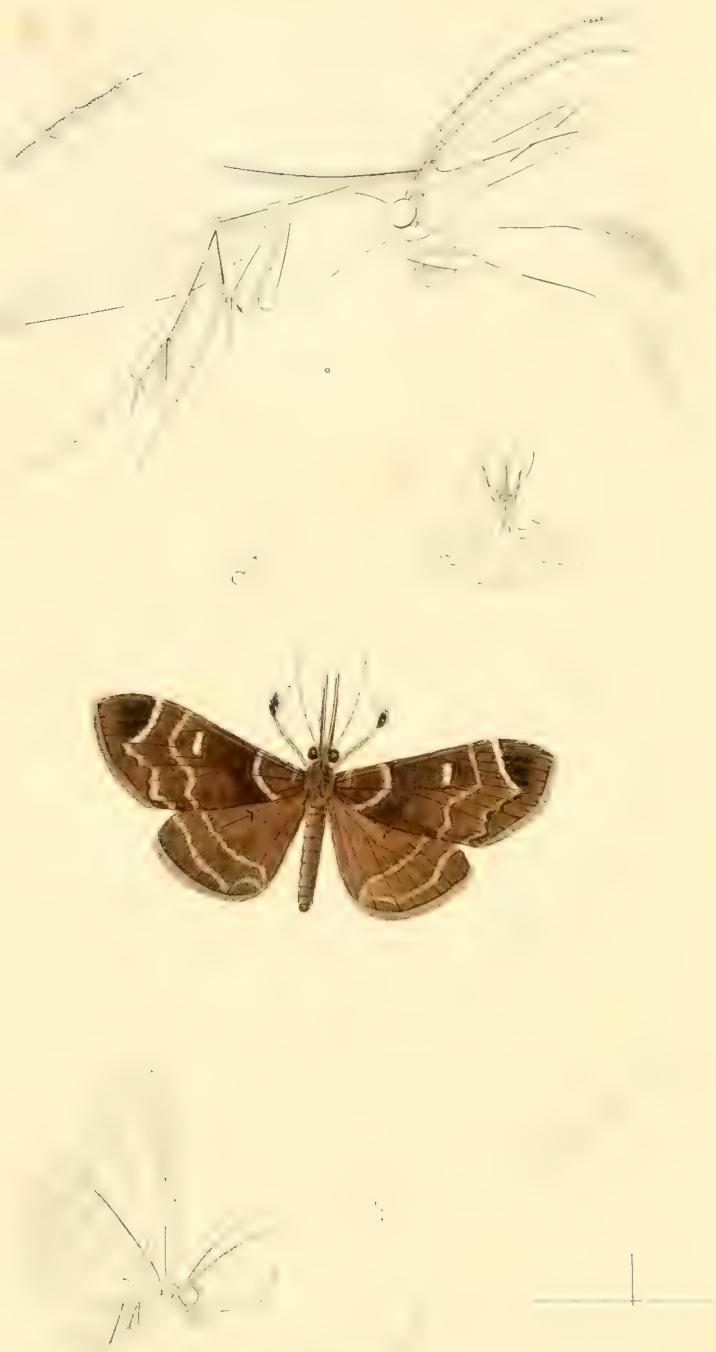

PARTRA 


\title{
NUM.
}

\section{Mastigophorus Parra, Poey.}

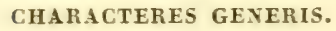

Gen. Mastigophorus, Poey.-Palpi maris longissimi, articulis tribus fere rqualibus, singulis longitudine abdominis, ultimo medium corporis attingente; primo producto, secundo et tertio in dorsum rejectis, prie se cubitis similitudinem prebentibus; freminæ breviores, producti, secundo longo, tertio paululum recurvo. Cæteris characteribus non differt ab Herminiis Latreillii.

Genre Mastigophore. - Palpes très-longs, dans le màle, à trois articles égaux, chacun de la longueur de l'abdomen, le dernier dépassant le corselet, le premier arancé, le second coudé et replié, arec le troisième, sur le dos; plus courts dans la femelle, à trois articles, dont le deuxième plus long, le troisième un peu recourbé. Tous les autres caractères de l'insecte parfait sont les mèmes que ceux des Herminies de M. Latreille.

Alis fuscis, strigis duabus flarescentilus; anticarum baseos altera extrorsum nigro repanda. puncto medio flavo et apice niģricante. 
Mastigophore Parra. - Ailes fauves, avec deux lignes d'un brun clair; une autre vers la base des supérieures, bordée de noir en dehors, ainsi que celle du sommet, et de plus un point central jaunâtre.

DESCRIPTION.

Voyez, dans la planche ci-jointe, le mâle de grandeur naturelle, ainsi que les deux sexes grossis, et le mâle beaucoup plus grossi encore, pour en faire connaitre les palpes, les antennes et les pattes.

Les palpes du mâle ont le dernier article garni en dessous de longs cheveux, qui partent de la base, et s'adossent à l'article jusqu'à l'extrémité. La touffe de poils, qu'on remarque aux jambes antérieures, est noire en dehors, et blanchâtre en dedans. La femelle n'a point de poils aux pattes; ses palpes ont la forme ordinaire de ceux des Herminies. Le dessous des ailes est pointillé de gris.

\section{OBSERVATION .}

Sur re Genre. - Le mot Mastigophore vient de deux mots

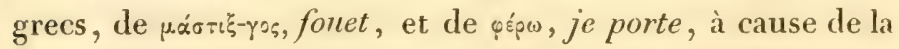
forme des palpes du mâle, qui ressemblent au fléau avec lequel on bat le blé dans les campagnes. Les Romains donnaient ce nom à des licteurs armés de verges pour surveiller les jeux publics.

HISTOIRE.

J'ai toujours pris cet insecte dans l'intérieur des appartements; il y est attiré par la lumière des lampes, et se repose 
souvent contre le mur, dans un port dailes indiqué par li figure. J'en ai apporté plusieurs femelles et un seul màle. De tous les papillons connus, c'est bien celui qui a les palpes les plus longs; car ceux des Libythées et des Herminies n'atteignent pas même au tiers de la longueur de ceux-ci. C'est ce qui en fait un genre extrèmement intéressant; et il est facile de se le procurer, car il est assez commun à la Havane. Jai brisé l'unique individu qui me restait, pour mieux faire mes dessins; mais je l'ai montré aupararant à MIII. Latreille, Boisduval, Duponchel, Lefebvre et Rambur.

Je me fais un devoir de dédier cette espèce à M. Ant. Parra, qui ma précédé dans la description et liconographie des animaux de lile de Cuba : car il a publié, en $1-87$, une Histoire naturelle des Poissons et des Crustacés de cette île. Cet ouvrage lui fait d'autant plus d'honneur qu'il a été imprimé et gravé à la Havane, dans un temps où il n'y avait qu'une seule imprimerie pour le journal officiel du Gouvernement.

Avril 1832. 


Gen. Syllepis, Pocy
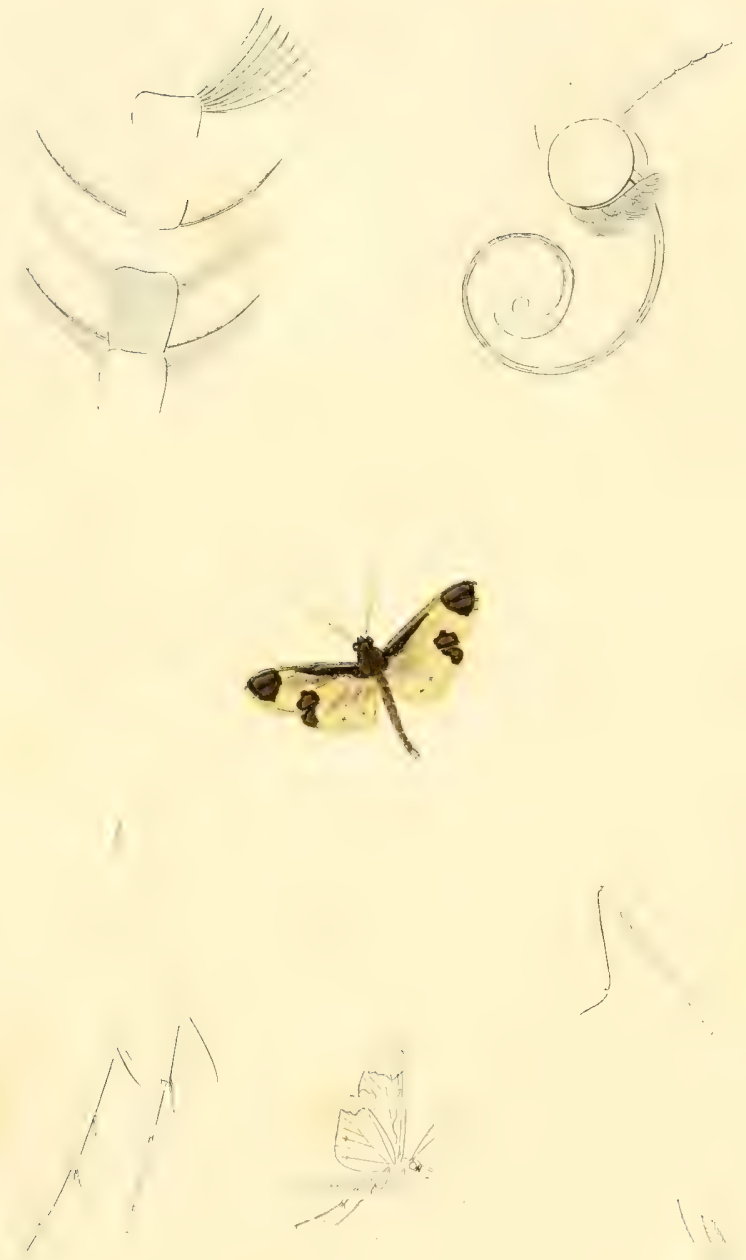

MIARIATISS 


\section{NUM.}

\section{Syllepis Marialis, pocy.}

CHARACTERES GENERIS.

Genus Srllepis, Poey. - Characteribus jam notis Pyralidum Linnæi Botydumque Latreiliii adde antennas pectinatas, saltem in mare, quia freminam non vidi; abdomen longissimum.

Genre Syluépide. - Ge genre a tous les caractères ordinaires du genre Pyrale de Limné, Botys de Latreille, sauf que les antennes sont pectinées, du moins dans le màle, car je n'ai pas vu la femelle. L'abdomen est plus long que dans les Pyrales ordinaires.

CHARACTERES SWECIEI.

Alis flavissimis, nitidis; anticis costa angulisque, posticis apice punctisque duobus, nigris.

Syléépide Mariatis. - Ailes d'un jaune vif un peu brillant; les supérieures ayant la plus grande partie de la côte et les angles extérieurs; les inférieures, le sommet et deux points, noirs.

\section{IESGRIPTION.}

Voyez le mâle dans la planche ci-jointe, avec des grossissements an trait. 
Les antennes, vues à la loupe, ont deux rangées d'écailles sur chaque article; ceux-ci supportent à la base deux branches latérales, ornées d'un double rang d'épines, et à l'extrémité, un faisceau d'écailles allongées qui recouvrent les branches de l'article suivant, à manière de toit. Les palpes sont noirs en dehors.

Les ailes sont d'un jaune un peu brillant; les taches brunes ont au centre des prunelles bleues métalliques; les deux petits points des inférieures qui sont bordés en arrière d'un peu de blanc luisant, ainsi que la bande brune des supérieures, vers la base. Le dessous est semblable au dessus, mais plus décoloré, et les taches brunes n'ont pas d'éclat.

OBSERVATIONS.

Sur LE GEnre.-Je ne connais que cette seule espèce, qui est le type du genre. Le mot Syllepis vient de deux mots grecs : de oùv, avec, et de rsxis, écaille. J'ai voulu exprimer le faisceau d'écailles allongées qui recouvrent et protégent les branches dont j’ai parlé. Il n'est pas cependant essentiel que ces écailles se retrouvent dans d'autres espèces pour les classer dans le même genre; il suffit que les anternes soient pectinées.

Sur l'espèce._Elle est assez voisine de Concordalis (Hüb.), mais toutefois facile à distinguer.

IISTOIRE.

Prise une seule fois à la Havane, sur les murs d'un appartement. Dans le repos, elle porte les ailes presque étendues et l'extrémité de l'abdomen relevée.

Avril 1832. 

Gen. Prvalis, tin .

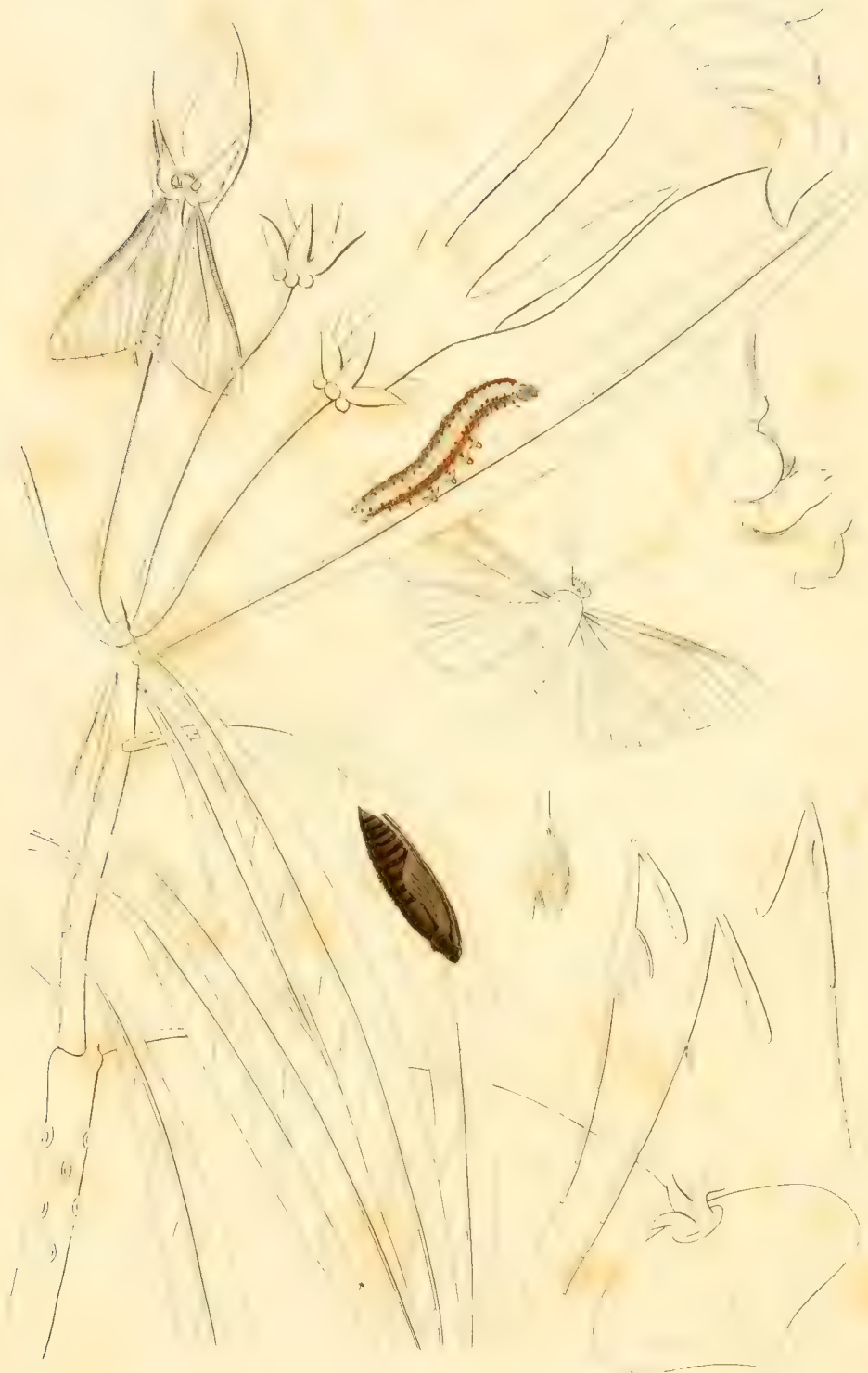

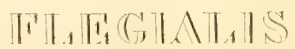




\section{NUM.}

\section{Pyralis Flegialis.}

Flegia, Cram., I40, D. Surinam.

Alis albis, costa anticarum cærulea.

Pyrale Fuegialis. - Ailes blanches, avec la côte des antérieures bleue.

DESCRIPTION.

Voyez, dans la planche ci-jointe, l'insecte parfait au port d'ailes et aux ailes étendues; les palpes, les trois premiers articles des antennes et les pattes, grossis; la chenille, la plante qui lui sert de nourriture; la chrysalide avec si coque, et le paquet de feuilles qui la cache. La plante a été copiée d'après l'Atlas du Dictionnaire d'Histoire naturelle de M. Levrault, et une figure du P. Plumier, combinés entre eux.

Les ailes sont d'un blanc luisant, avec un reflet violet vers l'extrémité des antérieures; la base de ces mêmes ailes et toute la surface des postérieures sont couvertes de longs poils blancs. Deux points noirs sur la tête, au devant des yeux, et deux autres sur chaque palpe; tout le reste du corps est blanc. Les pattes postérieures ont les jambes garnies de poils entre les deux paires d'ergots; ces poils sont noirs en dedans. Le crin du mâle est simple et retenu par un frein, qui part de la seconde cellule interne. Je n'ai pas la femelle sous les yeux.

La chenille a, sur le dos, deux rangées de points noirs et luisants, et une autre au dessus des stigmates; ces points servent de bordure à la bande rouge latérale. 
Sun LE Genre.-Quelle que soit l'autorité de Fabricius, je ne puis m'empêcher de le blâmer d'avoir bouleversé la nomenclature linnéenne dans les dernières familles de Lépidoptères. Le genre Pyrale, détourné de son acception primitive, et appliqué aux Tordeuses de Linné, supprimait, d'un seul trait, deux genres de ce célèbre naturaliste : double attentat de la part de son élève, l'un contre le savant, et l'autre contre la science. C'est pour cela que M. Latreille a établi le genre Botys, qui n'est autre chose que le genre Pyrale de Linné. A l'exemple des savants et modestes auteurs du Catalogue des Lépidoptères des environs de Vienne, de Jacob Hübner, dont le mérite n'est pas borné à celui d'avoir été le premier des iconographes, et de M. Duponchel dans son excellent ouvrage des Lépidoptères de France, j’ai cru devoir rétablir le genre linnéen, et respecter la priorité dans le grand homme, comme je l'aurais respectée dans le plus mince auteur.

Sur l'Espèce. - La figure de Cramer est si mauvaise, que les amateurs me sauront bon gré de leur avoir fát connaître non-seulement la chenille et la chrysalide, mais encore l'insecte parfait.

Malgrél'usage de terminer en alis tous les noms de Pyrales, je ne me serais point permis de faire ce léger changement au nom de Cramer, s'il n'y avait déja une espèce de papillon diurne, nommé Phlegia, dans la famille des Héliconiens.

\section{HISTOIRE.}

La femelle dépose ses oufs sur un arbre dont les qualités malfaisantes sont bien connues; c'est l'arbre des tombeaux, c'est encore trop souvent l'arbre de la vengeance (I), l'Ahouai

(I) Remarque communiquée par M. Moreau de Jonnès, auteur d'une Flore des Antilles manuscrite, et d'excellents ourrages sur leur histoire naturelle et physique. 
des Antilles, ou le Cerbera Thevetia de Linné, vulgairement appelé, à la Havane, Cabalonga. Les nombreuses chenilles qui en sortent dévorent les feuilles avec une étonnante rapidité. L'arbre, entièrement dépouillé en peu dejours, ne peut leur servir d'asile, ni les protéger dans leur dernier changement. C'est alors qu'elles errent à l'aventure, et vont se cacher sous les feuilles des arbres de toute espèce qui les environnent. L'olsservateur trompé, les retrouvant partout, croit au premier coup d'oil que la chenille est polyphage; mais bientôt il est éclairé sur la véritable cause de cette transmigration, en voyant l'Ahouai, si beau naguère, dépourvu en ce moment de feuilles et de fleurs.

Les mouvements vifs et rapides, la course légère, sont des facultés qui s'unissent rarement à la voracité dans les animaux. Aussi remarque-t-on que la chenille de cette espèce est plus lourde que toutes celles du même genre. Elles sont loin d'atteindre à l'agilité des Pyrales Verticalis et Urticata de Linné, si communes en Europe. Il est vrai que, dans nos climats froids, ces deux chenilles sont plusieurs mois à croître, passent même l'hiver dans leur premier état, et ne se transforment qu'au printemps de l'année suivante; tandis que, dans le brillant climat de l'île de Cuba, la naissance de l'insecte, ses développements successifs et sa dernière transformation s'opèrent seulement dans quelques jours.

Mais si l'augmentation de nourriture ralentit leur marche, elle n'affaiblit pas l'instinct qui les dirige dans les moyens de se mettre à couvert contre leurs ennemis, surtout contre les oiseaux, dont les yeux sont trompés par l'industrie des chenilles. Elles se cachent sous des feuilles, qu'elles savent lier avec tarit de précaution qu'il n'est pas facile de s'en apercevoir sans une attention particulière. Le nombre de feuilles qu'elles emploient varie selon leur forme et leur grandeur; celles du sureau sont toujours au nombre de trois; celles du citron, au nombre de deux; celles de l'Ahouai, en très-grand nombre. Les fils qu'elles rassemblent forment 
un tissu très-lâche, qui paraît destiné plutôt à retenir les feuilles qu’à rendre impossible l'attaque de quelques ennemis, qui, parce qu'ils sont plus petits, n'en sont que plus dangereux.

De ce nombre est une mouche, proprement dite, que M. Robineau-Desvoidy a étudiée dans ma Collection, et à laquelle, d'après ses mocurs, il a donné le nom spécifique de Botivora. La femelle de cet insecte n'est point guidée par la vue, et les ruses de la chenille sont inutiles contre elle. La mouche la découvre par l'odorat, et s'introduit au dessous des feuilles à travers le tissu trop lâche dont jeviens de parler; déposant alors ses oufs sur la peau de la chenille, elle attache à son corps le germe de prochaines douleurs et d'une mort certaine.

Je ne suivrai point ces nouvelles larves dans leur naissance et leur développement; ce serait faire l'histoire générale des chenilles dévorées par des mouches. Mais j'écris pour toute sorte de personnes, même pour celles qui n'ont point étudié l'histoire naturelle des insectes, et qui jetteront les yeux sur ce livre, à cause de l'intérêt que leur inspire tout ce qui a rapport à l'histoire de l'ile de Cuba. C'est pourquoi j'ajouterai que ces malheureuses chenilles deviennent la proie des larves que la mouche a déposées sur leur corps, et que, si elles conservent encore assez de vie pour se changer en chrysalide, elles n'arrivent jamais à l'état d'insecte parfait. Le nombre des victimes est si grand que, malgré l'extrême abondance des chenilles, le papillon devient rare; et, certes, nous n'en serons point étonnés, si nous considérons que tous les êtres doivent vivre et se perpétuer dans la nature, et que, parmi les êtres végétaux, l'Ahouai aurait enfin disparu de l'Amérique, si de nombreuses générations de Pyrales eussent toujours dévoré ses feuilles. 



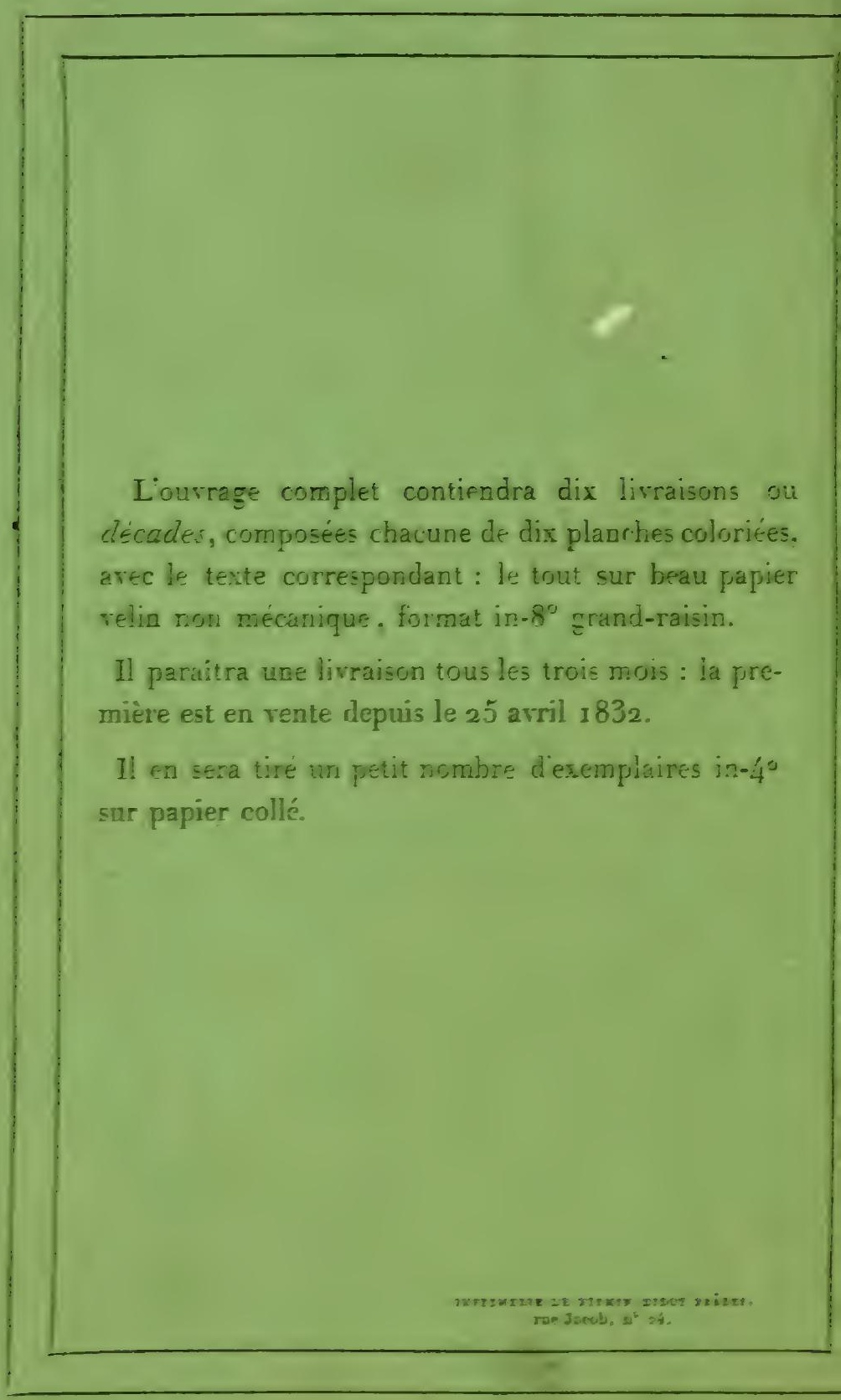




